$\$=-$ 国

\title{
Detection of micro aneurysms automatically for retinopathy screening
}

\author{
Josline Elsa Joseph ${ }^{1 *}$, R. J. Hemalatha, A ${ }^{1}$, Josephin Arockia Dhivya ${ }^{1}$, T. R. Thamizhvani ${ }^{1}$, R. Chandrasekaran \\ 1, G. Srividhya ${ }^{1}$ \\ ${ }^{1}$ Department of Biomedical Engineering, Vels Institute of Science, Technology and Advanced Studies , Pallavaram, Chennai 600117, \\ India \\ *Corresponding author E-mail: a.dhivya.se@velsuniv.ac.in
}

\begin{abstract}
Diabetic retinopathy is one among the severe diseases of eye leading to irreversible damage when left undiagnosed. Hence, robust automated system for diagnostic medical imaging is becoming a vital necessity in the healthcare industry. Microaneurysms are the first confirmed visual signs of onset of diabetic retinopathy. This work exploits the benefits of tristate median filtering and power law transform for improved candidate extraction work. It includes processing the image and normalization of intensities as microaneurysms are seen as low contrast and tiny.Tristate median filtering removes unwanted noise that would have added up while acquiring images. Power law transformation is applied to increase brightness. And makes the microaneurysms clearer in the image. The second approach involves extraction of retinal vessels from the image as miniature vessels can be falsely detected as candidate lesions. Weapply simple and effective morphological operations followed by detection of lesions by extended minima transform. Candidate features are extracted and then classified by K nearest Neighbour classifier.The performance of the proposed work is analysed giving accuracy specificity, and sensitivity values $91.5 \%, 82 \%, 93 \%$ respectively.
\end{abstract}

Keywords: Diabetes; Micro aneurysms; Retinal Vessels; Morphological Operation; Eye Fundus Image.

\section{Introduction}

Diabetic retinopathy is a chronic device which has caused a large number of people diagnosed by diabetic retinopathy will increase to 191 million by 2030 if appropriate measures are not carried out for its diagnosis. [1] It is largely seen in patients affected with diabetes for prolonged years. [2]

Diabetes mellitus damages even the miniature blood vessels and causes harm to retina. Hence it is important to diagnose diabetic retinopathy at the earliest. There are a various signs and symptoms which confirms the occurrence of diabetic retinopathy. The presence of microaneurysms is primarily considered as the first visible sign for the onset of the disease. Theprecautionary measures includeperiodic screeningwhich can recognise any minute chances for occurrence of this serious illness and can help prevent its progression .Robust and reliable image processing tools and detection systems are mandatein the field of image processing for precise judgement about verifying the disease. Microaneurysm Lesion isa reddish circular pattern with a diameter $<125 \mu \mathrm{m}$. [3-4]

Microaneurysms are seen as miniature low contrast components in the stereo funds image which may stand as a challenge its accurate detection. The progression of diabetic retinopathy is marked by the number of lesions in the fundus image .Diabetic retinopathy (DR) condition is classified as proliferative and non-proliferative DR. Non proliferative diabetic retinopathy further is classified into several subcategories based on the increasing count of lesions as mild, moderate and severe non proliferative diabetic retinopathy respectively. NDR leads to leakage of fluid or blood in the retina leading to retinal deposition and swelling. PDR occurs due to the formation of new vessels in the retina leading to many complications like tissue scarring, blocked vision and even blindness.

Further, the presence of retinal vessels largely produces false negatives due to misinterpretation works are not appropriate. The detection process is even more complicated when the microaneurysms displays diverse shades of the colour red. This can occur due to varying retinal images mainly due to varying illumination, pigmentation or presence of choroid in eyes. Haemorrhages can also be confused as microaneurysms adding to other complications like deposition of exudates.

\section{Literature review}

Several works prevail in the field of diabetic retinopathy screening by microaneurysm detection. In [5], an approach of multi scale coefficient method is carried out combined with dynamic thresholding for candidate extraction. This work has been applied on ROC and DIARETDB1 databases. In [6], candidate lesions are identified by a set of morphological reconstruction and fractal estimation of retinal vessels extraction afterpre-processing.Many works adopts image enhancement for easier detection of MAs [7]. In [8],CLAHE has been used in detailed manner for extraction of region of interest(microaneurysms).Template matching methods and their variants have been used by various authors using the lesion shape property matching Gaussian and other filters[9-13].In [14] the author adopts Circular Hough Transform to detect the centre point of Microaneurysm. Several Morphological operations based candidate detection is seen in [15-18].Double ring filtering is employed in [19] to detect microaneurysms. Intensity information of the inner and 
outer ring aids candidate detection, the results of which are classified by ANN. In $[20,21]$ lesions are classified by Bayesian classifier. Artificial neural networks and its variants have been employed by many authors for better classification approaches. [22], [23].

\section{Preprocessing}

The main aim of pre-processings to clear the image of noises and various image artefacts. The green channel of the red green blue components of eye fundus image is extracted as it provides finer details of eye compared to other components [24].The local contrast is also high in the green channel of the image. Tristate median filtering is employed on the image which prevents over segmentation thus avoiding erosion of edges and boundaries. Power law transformation brightens the image and provides better contrast. Vessel removal is done by effectively by Morphological operations and lesion detection by extended minima transform followed by feature extraction and classification by KNN classifier. The below fig .1 provides the detailed block diagram of our proposed work.

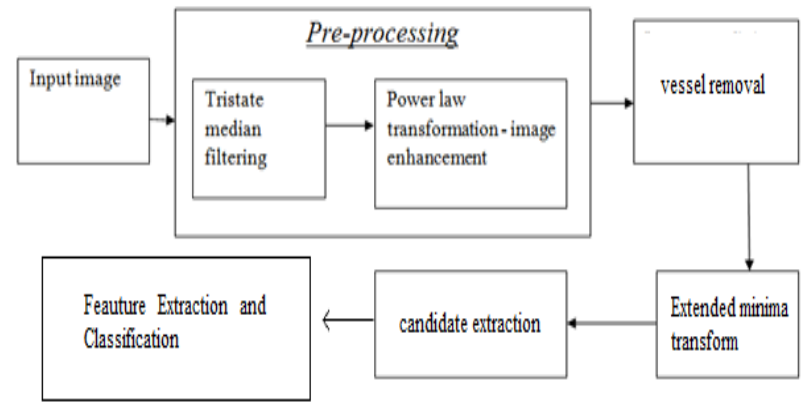

Fig. 1:

a) Tristate median filter

It is a combination of standard median filter and centre weighted median filter where the noise is detected by a customised impulse detector. It correlates the centre pixel value with the combination filter. It works as the name depicts deriving a three state decision. The regional noise is fixed by an adaptive process based on the tristate decision criteria. The pixels are thresholding the three states namely original pixels free of noise, second state of standard median filter schemed as corrupted pixel and centre weighted pixel selected as uncorrupted pixel. The main advantage of tristate median filter that pixels undergo a series of decision based scrutiny before it is marked as noise and filtered out.

The threshold $\mathrm{T}$ defines the three states is given in the Eq: 1 where a1,a2, a $3 \ldots$ represents pixels in the ascending order of intensity denoted as Aij

$$
\mathrm{T}=\left\{\begin{array}{l}
A i j \\
B i j \\
C i j
\end{array}\right.
$$

$\mathrm{T}>\mathrm{m} 1$

$\mathrm{m} 2 \leq \mathrm{T} \leq \mathrm{m} 1$

$\mathrm{T}<\mathrm{m} 2$

Bij - output of standard median filter

Cij - output of center weighted median filter

M1denotes the difference between the pixel considered and $\mathrm{Bi}$

M2denotes the difference between the pixel considered and $\mathrm{Cij}$

b) Power law transformation enhancement

It is a spatial domain filtering technique that functions by directly operating on the pixels and improves the contrast. The basic equation is given in Eq: 2. Where P \& $\mathrm{r}$ denotes output and input pixels respectively and $\mathrm{C}$ is the positive gamma constant.

Plaw $=$ Cry
The gamma value can be corrected based on the source image and its applications. When gamma value is greater than [1], the fundus image appears darker and when the value is lesser than 1, the image is illumined. Unlike other advanced enhancement techniques which makes the evaluation procedure longer and complex, this enhancement technique portrays the several possible lesions on the filtered image. Gamma correction enables to adjust the brightness based on the images available for study. Initially a trial test was carried out with values ranging from $0.4,0.6,0.8,1,2,3,4$ and 5.As the values are higher, the image projects the optic disc accurately, darkening other features in the image. When the value is lower than 0.8 , the image is over illumined. For the given database, enhancement works better when gamma $=3$

c) Vessel elimination

A set of simple morphological operations are carried out to eliminate vessels.Its robustness is important to remove even the miniature vessel structure. A closing operation is applied on the image after enhancement (Ip) using structuring element disk as lesions are also circular in shape. The next step is to take the difference of the closing image and enhanced image. Finally Otsu based thresholding is done to the binary image developed by vessel subtraction(VE) with the background image IC .This morphological vessel removal eliminates all unwanted pixels and shattered lesions paving way for accurate candidate extraction.

$\mathrm{VE}=\mathrm{IC}-\mathrm{Ip}$

\section{Candidate lesion extraction}

Morphological operator called extended minima transform is employed on the contrast enhanced image to determinate microaneurysms. They appear reddish brown and are tiny in the superficial retinal layers. These swelling like sacs can rupture and leak blood. Extended minima transform performs regional minima to the $\mathrm{h}$ minima transform. Here, the term minima refers to the minimal intensity pixels in the image .Regional minima is computed by selecting pixels with constant intensity value. The edge pixels are higher than the intensity value chosen. Extended minima transform computes the regional minimum values of a particular neighborhood and given threshold value given in Eq 3. Since our region of interest is low contrast, this transformation is beneficial to find out the minimal values in the image. The remaining pixels are rounded to zero.

Io $=$ EMT $(t$, Ie $)$

Where Ie is the power law transformation applied to enhance the image.

The result of extended minima transformation is a binary image consisting of white pixels representing the lesions in the input image. The threshold value $t$ decides which pixels to be eliminated. Hence, it is important to choose it effectively. We use a trial and error method to a range of values given below and analyse the images before confirmation.

$\mathrm{t} \in[0.01 .0 .02,0.03,0.04,0.05,0.06]$

Threshold value chosen here is 0.03 as it correctly suppresses the minima pixels and detects the pixels.Power transformation enhancement has effectively projected the component features in the image which works well for extended minima transformation to select the low intensity pixels. The difference between extended minima transformed image and vessel extraction image shows microaneurysm lesions. These lesions are validated by extracting specific features.

a) Feature extraction

The next step of our work is feature extraction of the microaneurysms detected. We have considered 7 features for classification as listed below. 
- $\quad$ Area (A) - It is estimated by the iterative calculation of pixels in the image. The area is found to be lower for true Microaneurysms.

- $\quad$ Perimeter - measures the length of external boundary of the microaneurysm candidate.

- Roundness - It shows how circular is the candidate lesion Denoted as $\mathrm{R}$, where $\mathrm{A}$ and $\mathrm{p}$ are area and perimeter respectively as given in eq 5

$\mathrm{R}=\frac{4 \pi \mathrm{A}}{\mathrm{P}^{2}}$

- Mean - The mean of each candidate considered is defined as the central value of a set of pixels with particular range.

$\bar{x}=\frac{1}{N} \sum_{i=1}^{N} X_{i}$

Standard deviation of each candidate as in Eq. 7.

$\mathrm{SD}=\left[\frac{1}{N-1} \sum_{i=1}^{N}\left(X_{i}-\bar{x}\right)^{2}\right]^{1 / 2}$

- Major axis Length (2A) of the candidate lesion is given by $\mathrm{Eq} .8$

$2 \mathrm{~A}=\mathrm{c}+\mathrm{d}$

Where $\mathrm{c}$ and $\mathrm{d}$ are denotes the distance of any point in the eternal boundary to the focus.

- Minor axis length $2 \mathrm{~B}$ is given in Eq.9 as

$2 \mathrm{~B}=(c+d)^{2}+f^{2}$

Where $\mathrm{c}$ and $\mathrm{d}$ denotes the distance of any point in the external boundary to the focus $\mathrm{f}$.

\section{Classification}

Once the features are extracted, KNN classifier is adopted to classify the candidate lesions as true MAs or not.Instance based leaning commonly termed as $\mathrm{K}$ nearest neighbour, is a non-parametric classifier.It functions by carrying outcomparison between the data point values of training and test feature space to evaluate the nearest neighbours. The value of $\mathrm{k}$ is based on user selection and it becomes the backbone for the following classification works. The data point value is assigned to the class adopting nearest neighbour based on selected $\mathrm{k}$ value. It is effective and simple classifier whose output is taken as a class membership, where no exclusive training stage is required in the intermediate. This classifier is trained to detect true and false microaneurysms. Increased level of sensitivity to noise makes KNN effective and stable as far as other complex classifiers are concerned.

\section{Results and discussion database}

Images for this work are taken from ROC public database.[25].The images under study consist of 100stereo fundus images of which 50 are training images and another 50 images are testing samples taken from DR screening camp.We have taken the trainingset for our work and divided it as testing and training images equally. An expert ophthalmologist estimated microaneurysms to aid performance evaluation of the proposed work. Pre-processing aims to fairly manipulate the input image to aid for accurate candidate detection. The intervening noise and motion artefactscause hurdle to true classification. Pre-processing includes filtering, enhancement and vessel elimination. Candidate extraction work includes application of Extended Minima transform and Extraction of features . Classifier determines the correct MAs from the incorrect ones. The robustness of the system is by some performance measures given below. Fig : 2 shows the pre-processing and candidate lesion detection stages. Sensitivity: defines the how precisely all the microaneurysm lesions have been classified.

Specificity: defines how precisely the non Microaneurysm lesions have been classified.

Accuracy: defines the overall precision of the proposed system.

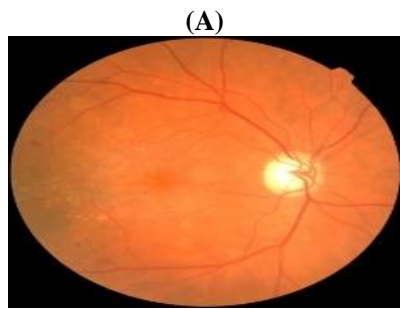

(B)

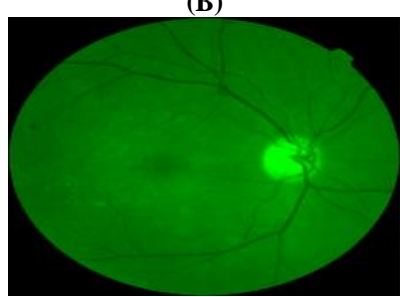

(C)

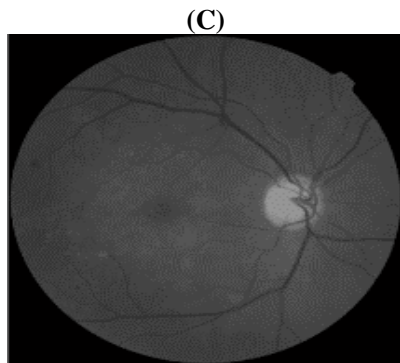

(D)

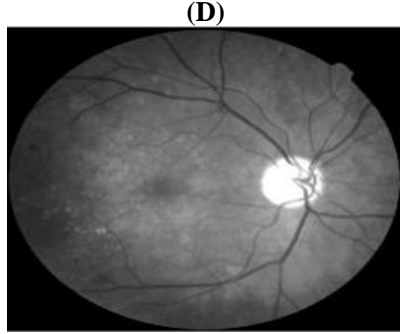

(E)
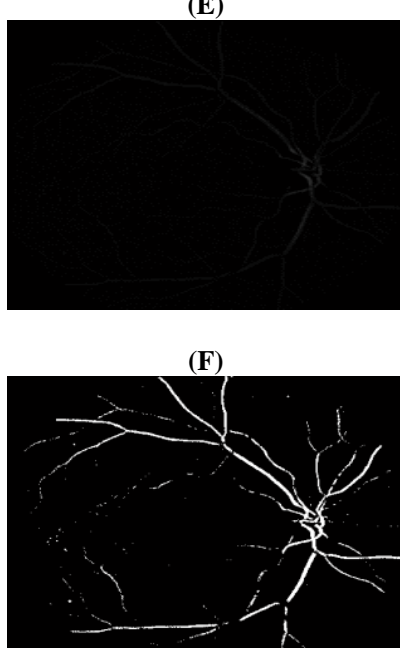

Fig. 2: (A) Input Image (B) Green Channel Extracted Image (C) Tristate Median Filtered Image (D) Power Law Transformation Enhanced Image (E) Vessel Subtracted Image (F) Vessel Extracted Image. 
The input image has been extracted of the green channel image where lesions are visible. The image noises are filtered effectively by Tristate Median filter. Power law transformation with gamma correction has been tested for values greater than and less than 1.The gamma value of 3 clearly depicts the microaneurysm like red spots and other illuminated lesions. The vessel elimination process employed here is very simple giving promising results. The vessel subtracted image is segmented by Otsu thresholding.Many bright lesions displaying high contrast as seen in the power law transformation enhanced image are also eliminated in the vessel elimination stage. Extended Minima Transform extracts low contrast lesions containing minimum intensities.It results in candidate extraction from the fundus image for further verification. The true microaneurysms are distinguished from false lesions by feature extraction and classification. The [7] features enable precise classification by KNN classifier. Fig 3 shows candidate extraction.
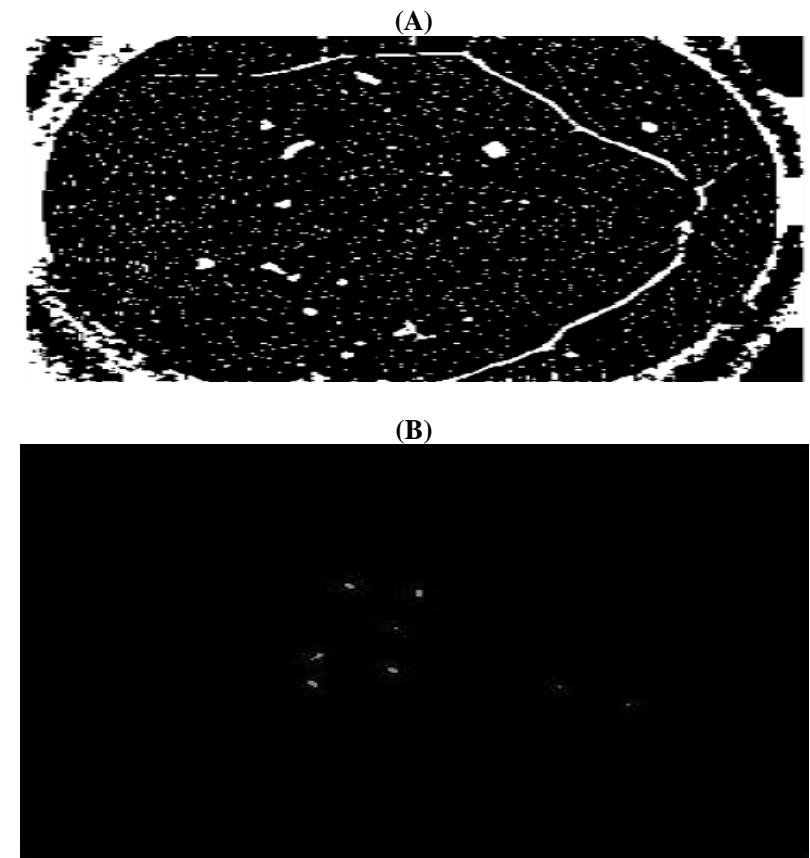

Fig. 3: (A) Extended Minima Transforms Image (B) Microaneurysms Detected.

(A)

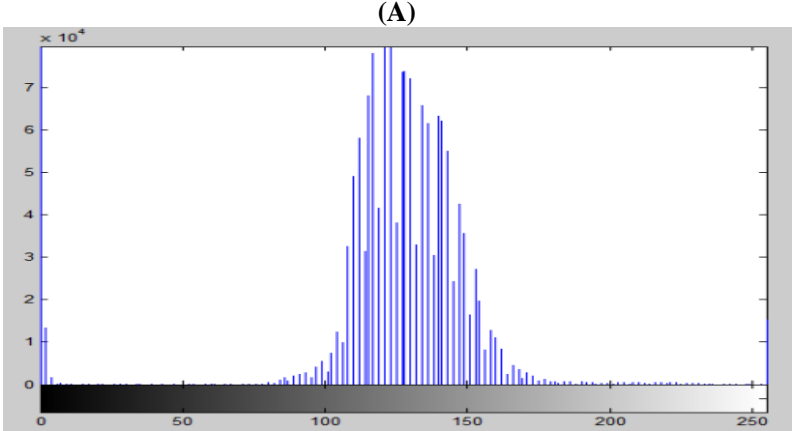

(B)

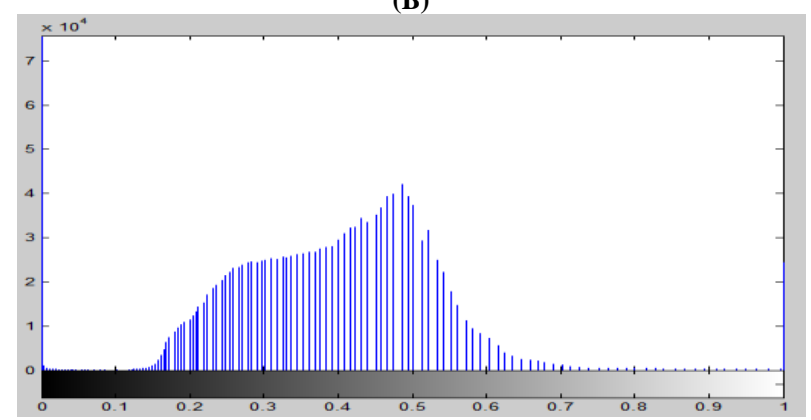

Fig. 4: (A) Histogram of CLAHE Enhanced Image (B) Power Law Enhanced.
The histogram of AHE , Power Law transformation and CLAHE Enhancement techniques given in Fig. 4, depicts that uniform distribution of intensities is better adopted by Power law transformation with corrected gamma value.

Table 1: Shows the Performance Evaluation Measures for KNN Classifier

\begin{tabular}{ll}
\hline Factors & Rates \\
\hline Accuracy & 91.5 \\
Specificity & 82 \\
Sensitivity & 93 \\
\hline
\end{tabular}

\section{Conclusion}

Diabetic retinopathy is one of the fast rising diseases which need to be screened in the earlier stages to prevent vision loss and blindness. In our work, a quality improved Algorithm for microaneurysm is proposed where the pre-processing sets itself on task to achieve higher levels of robustnessby adoption of tristate median filtering and power law transformation combined together. The ROI (microaneurysms) appear on the image after pre-processing techniques which eases the detection of candidate extraction. Extended minima transform effectively analyses lower intensities during candidate lesion extraction. A limited number of Featureextractions along with KNN make the proposed system simple to compute and less complex giving good results compared to other state of art methods. In future, other classification systems can be adopted and worked upon to for higher performance results.

\section{References}

[1] YingfengZheng, Mingguang He and Nathancongdon, 'The worldwide epidemic of diabetic retinopathy'. Indian Journal Of Opthalmology,vol 6, 2012,pg 428-431.

[2] Pedro RA, Ramon SA, Marc BB, Juan FB, Isabel MM, 'Prevalence And Relationship Between Diabetic Retinopathy And Neuropathy ;And Its Risk Factors In The North- East Of Spain, A Population Based Study.' Ophthalmic Epidemiol. 17(4) 2010, Pg 251-265.

[3] P.Massin,A.Erginayand A. Gaudric, ' Retinopathie Diabetique' ELSEVIER,SAS,2000.

[4] Mohandass G, Hari Krishnan G, Hemalatha R J , An Approach To Automated Retinal Layer Segmentation In SDOCT Images, International Journal of Engineering and Technology- UAE, vol. 2.25, 2018.

[5] B.Zhang, X.Wu,J.You,Q.Li, F.Karray, 'Detection Of Microaneurysm Using Multiscale Correlation Coefficients.' Patten Recognition, Vol43, 2010, Pp 2237-248.

[6] R.Roy,S.Aruchamy,andP.Bhattacharjee, “ Detection of retinal microaneurysms using fractal analysis and feature extraction technique, 'in proceedings of the 2 nd international conference on communication and signal processing (ICCSP'13),pp.469-471,Melmaruvathur,India April 2013.

[7] A.D. Fleming, S. Philip, K.A. Goatman, J.A. Olson, P.F. Sharp, Automated microaneurysm detection using local contrast normalization and local vessel detection, IEEE Transactions on Medical Imaging 25 (9) (2006) 1223-1232.

[8] N.S Datta,H.S.Dutta,M.De,nad S.Mondal,“An effective approach:image quality enhancement for microaneurysms detection of non-dilated retinal FUNDUS IMAGE,"Procedia technology,vol.10,pp.731-737,2013.

[9] The dynamic multiparameter template (DMPT) matching is applied for the detection of MAs that is more accurate as compared to conventional schemes. S.Ding and W.Ma,"An accurate approach for microaneurysm detection in 1851 , IEEE,stocklim, Sweden, august 2014.

[10] G. Quellec, M. Lamard, P.M. Josselin, G. Cazuguel, B. Cochener, C. Roux, Optimal wavelet transform for the detection of microaneurysms in retina photographs, IEEE Transactions on Medical Imaging 27 (9) (2008) 1230-1241. 
[11] I. Lazar, A. Hajdu, Microaneurysm detection in retinal images using a rotating cross-section based model. , 7906(1): 1405-1409, 2011.

[12] B. Zhang, X. Wu, J. You, Q. Li, \& F. Karray, Detection of microaneurysms using multi-scale correlation coefficients, Pattern Recognition, 43(6): 2237-2248, 2010.

[13] V. S. Hari, V. P. J. Raj, R. Gopikakumari, Quadratic filter for the enhancement of edges in retinal images for the efficient detection and localization of diabetic retinopathy, Formal Pattern Analysis \& Applications 1-21, 2015.

[14] Asadi S, Hassan. H, 'Detection of Micro aneurysms in Retinal Angiography Image using the circular Hough Transform', Journal of Advances in computer Research ,vol 3,No 1,2012,pages 1-12

[15] T. Walter, P. Massin, A. Erginay, R. Ordonez, C. Jeulin, J. Klein, Automatic detection of microaneurysms in color fundus images, Medical Image Analysis 11 (6) (2007) 555-566.

[16] T.Spencer, R. P. Phillips, P. F. Sharp, J. V. Forrester, Automated detection and quantification of microaneurysms in fluorescein angiograms, Graefes Archive for Clinical \& Experimental Ophthalmology, 230(230): 36-41, 1992.

[17] M. Niemeijer, G. B. Van, J. Staal, M. S. Suttorp-Schulten, M. D. Abràmoff, Automatic detection of red lesions in digital color fundus photographs, IEEE Transactions on Medical Imaging, 24(5): 584-92, 2005.

[18] A. D. Fleming, S. Philip, K. A. Goatman, J. A. Olson, P. F. Sharp, Automated microaneurysm detection using local contrast normalization and local vessel detection, IEEE Transactions on Medical Imaging, 25(9): 1223-32, 2006.

[19] A. Mizutani, C. Muramatsu, Y. Hatanaka, S. Suemori, T. Hara, H. Fujita .Automated microaneurysm detection method based on double ring filter in retinal fundus images. (2009), p. $72601 \mathrm{~N}$

[20] Sopharak, A., Uyyanonvara, B., Barman, S.: Automatic microaneurysm quantification for diabetic retinopathy screening. In: Proceedings of World Academy of Science, Engineering and Technology, p. 1722 (2013)

[21] Sopharak, A., Uyyanonvara, B., Barman, S.: Simple hybrid method for fine microaneurysm detection from non-dilated diabetic retinopathy retinal images. Comput. Med. Imag. Graph. 37(5), 394-402 (2013)

[22] T. Inoue, Y. Hatanaka, S. Okumura, C.Muramatsu, H. FujitaAutomated microaneurysm detection method based on eigenvalue analysis using hessian matrix in retinal fundus images Engineering in medicine and biology society (EMBC), 2013 35th annual internationalconference of the IEEE (2013), pp. 5873-5876

[23] ConfProc IEEE Eng Med Biol Soc. 2013; 2013:5873-6. doi: 10.1109/EMBC.2013.6610888. 'Automated microaneurysm detection method based on Eigenvalue analysis using Hessian matrix in retinal fundus images'.

[24] R. J. Winder, P. J. Morrow, I. N. McRitchie, J. R. Bailie, and P. M. Hart, 'Algorithms for digital image processing in diabetic retinopathy', Comput. Med. Imaging Graph. vol. 33, no. 8, pp. 608-622, 2009.

[25] University of Iowa. (2007) Retinopathy Online Challenge. [Online].http://webeye.ophth.uiowa.edu/ROC/var.1/www/. 